\title{
Study of very early phase GRB afterglows with MITSuME
}

\author{
Yoshihiko Saito $^{1}$, Yoichi Yatsu ${ }^{1}$, Hideya Nakajima ${ }^{1}$, Nobuyuki \\ Kawai $^{1}$, Katsuaki Asano ${ }^{1}$, Yu Aoki ${ }^{1}$, Mayumi Hayashi ${ }^{1}$, \\ Seongdeng Song ${ }^{1}$, Kosuke Kawakami ${ }^{1}$, Kazuki Tokoyoda ${ }^{1}$, \\ Takahiro Enomoto $^{1}$, Ryuichi Usui ${ }^{1}$, Daisuke Kuroda ${ }^{2}$, \\ Kenshi Yanagisawa ${ }^{2}$, Hiroyasu Shimizu ${ }^{2}$, Hiroyuki Toda ${ }^{2}$, \\ Shogo Nagayama ${ }^{3}$, Hidekazu Hanayama ${ }^{4}$, Michitoshi Yoshida ${ }^{5}$ \\ and Koji Ohta ${ }^{6}$ \\ ${ }^{1}$ Dept. of Physics, Tokyo Institute of Technology, \\ 2-12-1, Oookayama, Meguro, Tokyo, 152-8551, Japan \\ email: saitoys@hp.phys.titech.ac.jp \\ ${ }^{2}$ Okayama Astrophysical Observatory, National Astronomical Observatory of Japan, \\ Kamogata, Okayama 719-0232, Japan \\ ${ }^{3}$ National Astronomical Observatory of Japan, \\ 2-21-1, Ohsawa, Mitaka, Tokyo, 181-8588, Japan \\ ${ }^{4}$ Ishigaki Astronomical Observatory, National Astronomical Observatory of Japan, \\ 1024-1, Arakawa, Ishigaki, Okinawa, 907-0024, Japan \\ ${ }^{5}$ Hiroshima Astrophysical Science Center, Hiroshima University \\ 1-3-1, Kagamiyama, Higashi-Hiroshima, Hiroshima, 739-8526, Japan \\ ${ }^{6}$ Dept. of Astronomy, Kyoto University, \\ Kitashirakawa-Oiwake, Sakyo, Kyoto, 606-8502, Japan
}

\begin{abstract}
We review the results of very early phase optical follow-up observations of recent gamma-ray bursts (GRBs) with the multi-color optical telescopes "MITSuME". The MITSuME telescopes were designed to perform "real time" and "automatic" follow-up observations prompted by the GCN alerts via the Internet. The rapidly slewing equatorial mounts allow MITSuME to start photometric observations within 100 seconds after the trigger for several GRBs. In particular, we detected a brightening just after the trigger for two GRBs. These phenomena could be interpreted as the "on-set" of afterglow. In this paper we summarize these optical observations with a brief interpretation.
\end{abstract}

Keywords. GRBs, Afterglows, Optical

\section{Introduction}

Early phase variability in the optical afterglow of GRBs should provide some important information about the circumburst medium and, therefore, on the origin of GRBs.

The robotic telescope system MITSuME (Multicolor Imaging Telescope for Survey and Monstrous Explosions), prompted by the GCN alerts, can start observations within about a minute after the triggers. We have three optical telescopes in different regions in Japan (Akeno 0.5m, Okayama 0.5m, and Ishigakijima 1.05m). Each telescope has a tricolor camera that can take images in three different color $\left(g /\right.$ SDSS), $R_{C}, I_{C}$ (JohnsonCousins)) at the same time.

In the next section we report the results of the observation in which we could successfully monitor the light curve of the GRB afterglow. 

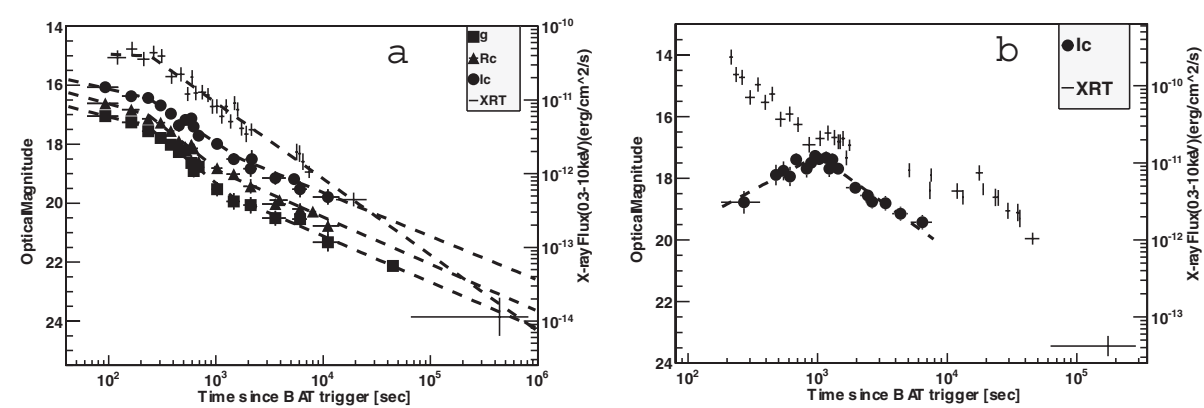

Figure 1. (a) The light curve of GRB090426. (b) The light curve of GRB100219A.

\section{Results of Observation}

In the last three years, we have successfully observed six GRBs. Brief reports for four of those GRBs are described as follows.

GRB090426. This GRB should be the most distant $(\mathrm{z}=2.61)$ short burst (Antonelli

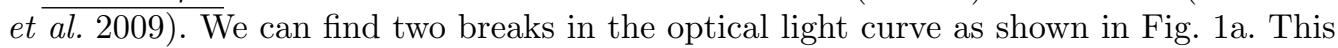
result implies two possible scenarios that are "Two component jet scenario" (Racusin et al. 2008) and "Multiple energy injection scenario" (Xin et al. 2011).

GRB091208B. This is a long GRB at $\mathrm{z}=1.06$. We find that the optical light curve has a simple power law decay until $T_{0} \sim 2000 \mathrm{sec}$. The energy spectral index of electrons could be estimated from the index of this power law. This result is consistent with the one estimated from the decay index of X-ray.

GRB100219A. Because this GRB has a large redshift $(\mathrm{z}=4.67)$, we could identify the optical counterpart only in $I_{c}$ band. We can follow the brightening phase in the light curve until $T_{0} \sim 1000 \mathrm{sec}$, while X-ray light curve appears to have a small bump around this time (Fig. 1b). The simultaneous optical and X-ray rebrightening could be explained as the onset of the afterglow. From the peak of onset, we derived that the initial Lorentz factor should be $\Gamma_{0} \sim 84$. Based on the optical brightening index, we also find that the ISM around the GRB should have an intermediate density profile between the ISM model and the wind model.

GRB100906A. Because the Akeno telescope of MITSuME could start the observation of this GRB 26 seconds after the trigger, we could obtain a very early phase light curve. This light curve shows a brightening phase until $T_{0} \sim 150 \mathrm{sec}$ and a power law decay thereafter. This optical onset suggests that the ISM around the GRB should be explained by a wind model and the initial Lorentz factor should be $\Gamma_{0} \sim 160$.

\section{References}

Antonelli, L. A., D’Avanzo, P., Perna, R., et al. 2009, A\&A, 507L, 45

Racusin, J. L., Karpov, S. V., Sokolowski, M., et al. 2008, Nature, 455, 183

Xin, L., Liang, E., Wei, J., et al. 2011, MNRAS, 410, 27 